\title{
Epidermal Growth Factor Receptor Mutations in Small Cell Lung Cancer Patients Who Received Surgical Resection in China
}

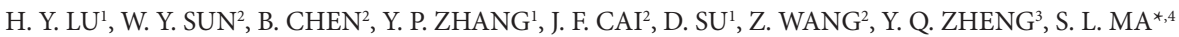 \\ ${ }^{1}$ Zhejiang Key Lab. of the Diagnosis \& Treatment Technology on Thoracic Oncology, Zhejiang Cancer Hospital, Hangzhou 310022, P.R. China; \\ ${ }^{2}$ Zhejiang Cancer Hospital, Hangzhou 310022, P.R. China; ${ }^{3}$ SurExam Bio-Tech Co. Ltd., Guangzhou Technology Innovation Base, Science City, \\ Guangzhou 510663, P.R. China; ${ }^{4}$ Hangzhou First People's Hospital, Hangzhou 310006, P.R. China
}

*Correspondence: shenglinma@163.com

Received June 20, 2011 / Accepted August 31, 2011

\begin{abstract}
To know the incidence of epidermal growth factor receptor (EGFR) mutations in small cell lung cancer (SCLC) patients who received surgical resection in mailand China. xTAG technology was used to detect the EGFR exon 19 and exon 21 mutations of 40 patients with SCLC who received surgical treatment in Zhejiang Cancer Hospital from 1998 to 2010.2 of 40 cases were found with mutations in exon 19 of the EGFR gene. The mutation in exon 19 of the EGFR gene is in a female and non smoking patient which pathology is SCLC combined adenocarcinoma, and the other is male and smoking patient which pathology is SCLC combined squamous cell carcinoma. The EGFR mutation is rare in SCLC patients, and EGFR mutation might occur more often in combined SCLCs than conventional patients.
\end{abstract}

Key words: EGFR, gene mutation, xTAG technology, SCLC

In recent years, mutations in the epidermal growth factor receptor (EGFR) gene have been reported to be present in nonsmall cell lung cancer (NSCLC) and related to response of tumors to EGFR tyrosine kinase inhibitors, suggesting its usefulness as a biomarker. However, there are few large studies reporting the mutations status of patients with small-cell lung cancer (SCLC). Since now, a case study about one Japanese patient with gefitinibresponsive SCLC has reported that this patient harbors a deletion in exon 19 of EGFR [1]. Moreover, another case study has also reported that one American SCLC patient with EGFR mutation who have never smoked is response to gefitinib [2]. In China, there is also one case report of patient with SCLC response to gefitinib, but the status of mutation is unknown [3].

More recently, a prospective study on 76 specimens from patients with SCLC between 2004 and 2009 at the National Taiwan University Hospital have repoted that 2 cases (2.6\%) tested positive for the EGFR mutation and both were deletions in exon 19 by reverse transcription polymerase chain reaction

Abbreviations: EGFR: epidermal growth factor receptor, SCLC: small cell lung cancer, RT-PCR: reverse transcription polymerase chain reaction, NSCLC: non small cell lung cancer, MFI: median fluorescence intensity, SSCP: single strand conformation polymorphism, ARMS: amplified refractory mutation system
(RT-PCR) and direct sequencing. Specimens in this report included 10 computed tomography-guided biopsy specimens, 17 echo-guided aspiration specimens, 37 echo-guided biopsy specimens, 1 surgical lobectomy specimen, and 11 malignant pleural effusion specimens [4]. Besides, a study of EGFR gene mutations occured in 122 Japanses patients with SCLC were evaluated. And EGFR mutations were detected in 5 SCLCs (4\%). All 122 patients were available for molecular genetic analysis included 102 specimens obtained by biopsy, and 20 from surgically resected tumors[5]. As a comprehensive analysis and incidence of mutations of SCLC in mainland China has not been reported, in present study, we detected EGFR exon 19 and exon 21 mutations of 40 SCLC patients by xTAG technology who were from Zhejiang Cancer Hospital. The specimen from surgey can better reflect the clinicopathologic status, and all of our specimens are from surgical resected tumors.

\section{Materials and Methods}

Patient characteristics. Between 1998 and 2010, 40 SCLC specimens were collected in Zhejiang Cancer Hospital, China. All 40 specimens obtained from surgical resected tumors. Histologic diagnosis of SCLC was based on the standard criteria defined by WHO classification. There are 5 female, 35 male. 
Age from 22 to 73 years old and median age is 54 years old. The stage: IA 4 cases, IB 3 cases, IIA 7 cases, IIB 5 cases, IIIA 19 cases and IIIB 2 cases by seventh edition of the TNM classification for lung cancer. Smoking history:non-smoker 7 cases, light smoker 3 cases, moderate moker 3 cases and heavy smoker 27 cases. Histological type: conventional type 37 cases, combined type 3 cases. Among the patients, 35 (87.5\%) were males, 33 $(82.5 \%)$ were smokers and $27(67.5 \%)$ heavy smokers.

xTAG technology for gene mutation. xTAG technology was used to detect the EGFR exon 19 and exon 21 mutations of 40 patients with SCLC who received surgical treatment in Zhejiang Cancer Hospital from 1998 to 2010. The xTAG technology contain the following basic steps: 1) amplify the regions of target genes by multiplex PCR (Table 1$)(\mathrm{H} 2 \mathrm{O}$ $13.8 \mu \mathrm{l}, 5 \times \mathrm{PCR}$ buffer $10 \mu \mathrm{l}, 25 \mathrm{mM} \mathrm{MgCl} 24 \mu \mathrm{l}$, dNTPs $2 \mu \mathrm{l}$, primers $10 \mu \mathrm{l}$, DNA polymerase $0.2 \mu \mathrm{l}$, sample $10 \mu \mathrm{l}, 94^{\circ} \mathrm{C}$ for 30 $\mathrm{s}, 52^{\circ} \mathrm{C}$ for $30 \mathrm{~s}$ and $72^{\circ} \mathrm{C}$ for $30 \mathrm{~s}, 30$ cycles); 2) digest by exonuclease and hydrolysis by alkaline phosphatase (exonuclease $0.5 \mu \mathrm{l}$, alkaline phosphatase $0.5 \mu \mathrm{l}, 2 \times$ buffer $10 \mu \mathrm{l}$, PCR product $9 \mu \mathrm{l}, 37^{\circ} \mathrm{C} 2 \mathrm{~h}$ ). The PCR mixture was treated using EXO-SAP to remove excess nucleotides and primers; 3 ) allele specific primer extension (ASPE) (Table 2) $(\mathrm{H} 2 \mathrm{O} 7.8 \mu \mathrm{l}, 10 \times \mathrm{PCR}$ buffer $2 \mu \mathrm{l}, 25 \mathrm{mM} \mathrm{MgCl} 0.5 \mu \mathrm{l}$, dNTPs (no dCTP) $1 \mu \mathrm{l}$, Biotin-dCTP $0.5 \mu \mathrm{l}$, primers $5 \mu \mathrm{l}$, DNA polymerase $0.2 \mu \mathrm{l}$, EXO-SAP-treated PCR product $3 \mu \mathrm{l}, 94^{\circ} \mathrm{C}$ for $30 \mathrm{~s}, 56^{\circ} \mathrm{C}$ for $1 \mathrm{~min}$ and $74^{\circ} \mathrm{C}$ for $2 \mathrm{~min}, 30 \mathrm{cycles})$. The EXO-SAP-cleaned PCR product subjected to an ASPE step. The Tsp DNA polymerase will extend only primers which are $100 \%$ match to the templates; 4) hybridization(beads $2 \mu$, hybridization solution $33 \mu \mathrm{l}$, ASPE product $5 \mu \mathrm{l}, 95^{\circ} \mathrm{C}$ for $3 \mathrm{~min}$, followed by $30 \mathrm{~min}$ incubation at $37^{\circ} \mathrm{C}$ ). The ASPE products were hybridized to specific anti-tag probes that were pre-coated on the polystyrene microspheres;

Table 1. The sequences of PCR primers

\begin{tabular}{llcc}
\hline Exon & Sequence, $5^{\prime} \rightarrow 3^{\prime}$ & PCR product length (bp) & Sensitivity, $\%$ \\
\hline 19 & CCAGAAGGTGAGAAAGTTAAAATACCCCCACACAGCAAAGCAG & 115 & 5 \\
21 & ACCGCAGCATGTCAAGATCACCCCTGGTGTCAGGAAAATGCT & 131 & 5 \\
\hline
\end{tabular}

Table 2. The sequences of ASPE primers

\begin{tabular}{|c|c|c|}
\hline Exon & Type & Universal tage - Primer, $5^{\prime} \rightarrow 3^{\prime}$ \\
\hline \multirow[t]{22}{*}{19} & Wt & СТTCTCATTAACTTACTTCATAATCTATCAAGGAATTAAGAGAAGC \\
\hline & $\Delta \mathrm{E} 746-\mathrm{A} 750(\mathrm{~K} 745: \mathrm{AAA})$ & TCAATTACTTCACTTTAATCCTTTAATTCCCGTCGCTATCAAAAC \\
\hline & $\Delta \mathrm{E} 746-\mathrm{A} 750(\mathrm{~K} 745: \mathrm{AAG})$ & СTTTTCATCAATAATCTTACCTTTTTCCCGTCGCTATCAAGACA \\
\hline & $\Delta \mathrm{L} 747-\mathrm{E} 749$ ins $\mathrm{P}$ & AAACAAACTTCACATCTCAATAATCGTCGCTATCAAGGAACCAA \\
\hline & $\Delta \mathrm{L} 747-\mathrm{A} 750$ ins $\mathrm{P}$ & TCATTTCAATCAATCATCAACAATCGTCGCTATCAAGGAACCAT \\
\hline & $\Delta \mathrm{L} 747-\mathrm{T} 751$ & TCAATCATCTTTATACTTCACAATCGTCGCTATCAAGGAATCT \\
\hline & $\Delta \mathrm{L} 747-\mathrm{T} 751$ ins $\mathrm{A}$ & TTACTCAAAATCTACACTTTTTCACCCGTCGCTATCAAGGCAT \\
\hline & $\Delta \mathrm{L} 747-\mathrm{S} 752$ & CTTTTTCAATCACTTTCAATTCATCCGTCGCTATCAAGGAACAT \\
\hline & $\Delta \mathrm{L} 747-\mathrm{S} 752$ ins $\mathrm{V}$ & CAATATCATCATCTTTATCATTACCCCGTCGCTATCAAGGTTC \\
\hline & $\Delta \mathrm{L} 747-\mathrm{S} 752$ ins $\mathrm{D}$ & AATCTACAAATCCAATAATCTCATCCGTCGCTATCAAGGATCC \\
\hline & $\Delta \mathrm{L} 747-\mathrm{S} 752$ ins $\mathrm{Q}$ & ATCAAATCTCATCAATTCAACAATCGTCGCTATCAAGGAACAG \\
\hline & $\Delta \mathrm{L} 747-\mathrm{S} 752$ ins $\mathrm{S}$ & STTCATAACTACAATACATCATCATCGTCGCTATCAAGGAATCG \\
\hline & $\Delta$ E749-S752 ins D & AAACTAACATCAATACTTACATCACTATCAAGGAATTAAGAGATCC \\
\hline & $\Delta \mathrm{E} 746-\mathrm{T} 751$ ins $\mathrm{T}$ & TCAAAATCTCAAATACTCAAATCATCCCGTCGCTATCAAGACC \\
\hline & $\Delta \mathrm{E} 746-\mathrm{P} 753$ ins VS & CTACTAATTCATTAACATTACTACCCCGTCGCTATCAAGGTCT \\
\hline & $\Delta \mathrm{E} 746-\mathrm{T} 751$ ins $\mathrm{Y}$ & AATCCTTTTTACTCAATTCAATCACCGTCGCTATCAAGGTATCA \\
\hline & $\Delta \mathrm{L} 747-\mathrm{T} 751$ ins $\mathrm{Q}$ & CTACAAACAAACAAACATTATCAACCGTCGCTATCAAGGAACAA \\
\hline & $\Delta \mathrm{E} 746-\mathrm{T} 751$ ins $\mathrm{A}$ & TAACATTACAACTATACTATCTACCCCGTCGCTATCAAGGCTT \\
\hline & $\Delta \mathrm{E} 746-\mathrm{A} 750$ ins $\mathrm{AP}$ & TATATACACTTCTCAATAACTAACTCGCTATCAAGGCCCCAAC \\
\hline & $\Delta \mathrm{L} 747-\mathrm{T} 751$ ins $\mathrm{V}$ & CTTTTCATCTTTTCATCTTTCAATCGTCGCTATCAAGGTATCT \\
\hline & $\Delta \mathrm{L} 747-\mathrm{S} 752$ ins $\mathrm{E}$ & ECTTTAATCCTTTATCACTTTATCACGTCGCTATCAAGGAACCG \\
\hline & $\Delta \mathrm{R} 748$ - $\mathrm{T} 751$ ins $\mathrm{LS}$ & TTACСTTTATACСTTTCTTTTTACCGCTATCAAGCTATCAAGCC \\
\hline \multirow[t]{5}{*}{21} & $\mathrm{Wt}(1)$ & TAATTATACATCTCATCTTCTACACAAGATCACAGATTTTGGGCT \\
\hline & $\mathrm{Wt}(2)$ & СТACTATACATCTTACTATACTTTGATTTTGGGCTGGCCAAACT \\
\hline & L858R (1) & СТTTTCAATTACTTCAAATCTTCAAAGATCACAGATTTTGGGCG \\
\hline & L858R (2) & CAATATACCAATATCATCATTTACAGATCACAGATTTTGGGCGT \\
\hline & L861Q & CTACATATTCAAATTACTACTTACGATTTTGGGCTGGCCAAACA \\
\hline
\end{tabular}




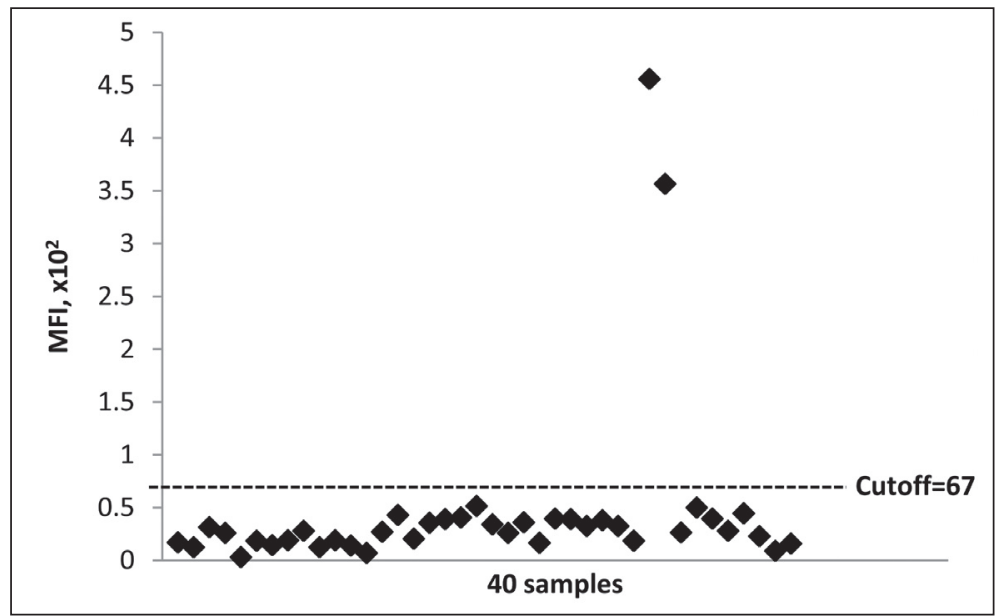

Figure 1. The figure showed that MFI of two samples are above the value of cut off.

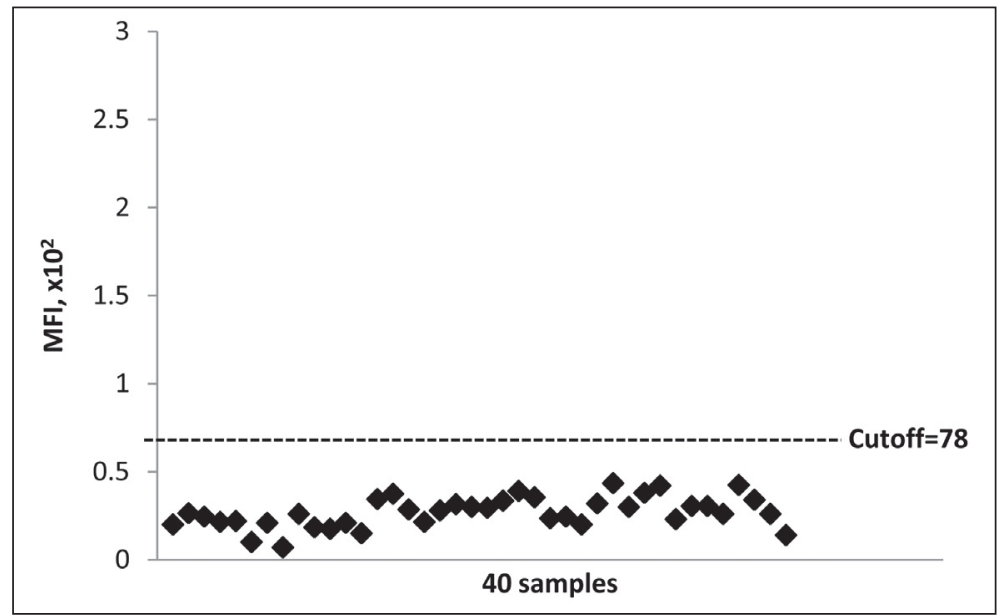

Figure 2. The result of EGFR E21 mutation in 40 cases. According to the figure, no sample has a value of MFI above the cut off, so no mutations were detected.

and 5) Luminex analysis. The beads were then applied to the Luminex 200 and median fluorescence intensity (MFI) read.

Data analysis. The statistical significance of mean values was determined by SPSS 13.0. A p value $<0.05$ was considered statistically significant.

Table3. Clinicopathologic features of SCLCs with and without EGFR mutations

\begin{tabular}{lcc}
\hline & Mutated & Wild-type \\
\hline No. of patients (total, N =40) & 2 & 38 \\
Age (median) & 62 & 54 \\
Sex (female/male) & $1 / 1$ & $4 / 34$ \\
$\begin{array}{l}\text { Smoking history } \\
\text { (median pack-years) }\end{array}$ & 23 & 30 \\
$\begin{array}{l}\text { Histologic type } \\
\text { (conventional/combined) }\end{array}$ & $0 / 2$ & $37 / 1$ \\
\hline
\end{tabular}

\section{Results}

Three patients were diagnosed with lung cancer that had combined small and non-small cell pathology, two showed EGFR mutation. Of the 40 examined SCLC specimens( Table 3 ), only 2 were found to be EGFR mutation positive. The EGFR mutation rate in SCLC was $5 \%$ in our cohort. The median age of the patients was 54 years old. Of the two patients with EGFR-mutated tumors, one was a male smoker and the other was a female never smoker. The mutations are all in exon 19: del E746-A750(K745 AAA) (Figure 1 and Figure 2).

Case 1. The first patient was a 61-year-old male heavy smoker who reported a chronic cough with chest tightness for over 20 days. Chest CT revealed a tumor mass in right hilar space. He received lobe surgey. Immunohistochemical stains revealed positivity of CHG-A/CgA, CD56 and synaptophysin. The pathological diagonosis is SCLC combined 


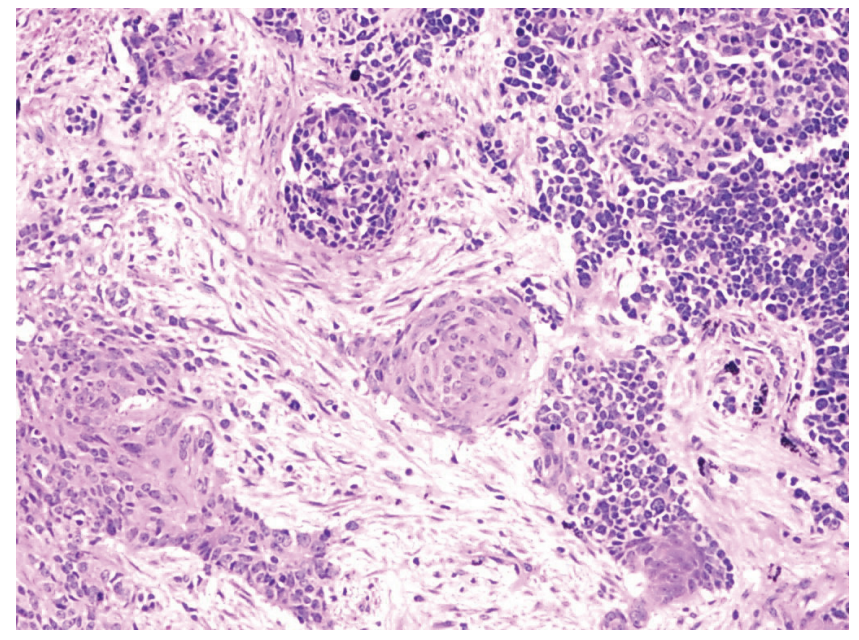

Figure 3. case 1:SCLC combined squamous cell lung cancer

squamous cell lung cancer (Figure 3 ). The initial TNM stage was T2aN1M0(IIA). He received three courses of chemotherapy with etoposide and cisplatin and lung metastasis appear three months after surgey and a progressive disease was obtained. Then he received four courses of docetaxel and endostar and a complete response was obtained. There is no relapse 16 months after surgey.

Case 2. The second patient was a 62-year-old female never smoker with no complain. Chest CT revealed a tumor in the upper right lung when received physical examination. She received upper-middle lobe resection and lymph node dissection. The pathological diagonosis was SCLC combined middle differentiation adenocarcinoma (Figure 4). The patient was diagnosed with limited-stage SCLC, and the stage is T1N2M0(IIIA) by TNM staging system. One month after surgey, brain metastasis developed. She received five courses of chemotherapy with irinotecan plus carboplatin and whole brain irradiation. Thirteen months after diagnosis, bone metastasis and lung metastasis developed. Then she received palliative radiotherapy and chemotherapy with four courses of irinotecan and endostar, and a stable disease was obtained. The patient is alive more than 22 months after diagnosis.

\section{Discussion}

Previous studies found that EGFR mutations were more frequent in NSCLC patients who were female, nonsmokers, and adenocarcinoma simultaneously. The incidence of EGFR mutation in NSCLC is more higher in China than in the United States and European countries [6-7]. However, the incidence of EGFR mutation in SCLCs in mainland China is still unknown. A high percentage of cases (28\%) showed that SCLC combinations with NSCLC, in detail, SCLC with large cell carcinoma was the most, followed by adenocarcinoma and squamous cell carcinoma [8]. EGFR mutations were

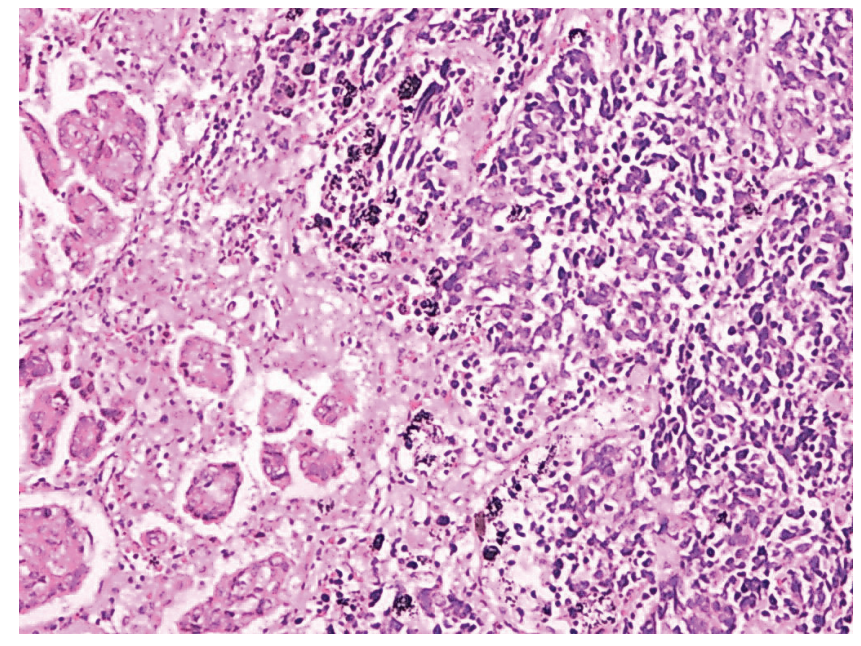

Figure 4. case 2: SCLC combined middle differentiation adenocarcinoma

detected in five SCLCs (4\%) in a study reported by Japanese and these five patients were mainly in the light smoker and histologic combined subtype. The specimen from surgey can better reflect the clinicopathologic features than biopsy and malignant pleural effusion. Most of the specimens were got from biopsy reported by Akiko Tatematsu in Japan [5], and only one specimen is got from surgey reported by Shiao $\mathrm{TH}$ in Taiwan [4]. Whereas, in our study, all specimens are got from surgey.

Besides, / the conventional method to test mutations is sequencing. As the somatic mutated DNA is usually masked under its wild-type (WT) DNA, it is very difficult to detect by sequencing. However, SurPlex-xTAG developed by SurExam is a very sensitive technology (1\%-5\% sensitivity), and allow for high throughput[9]. Thus, xTAG technology was used to detect the 24 most frequent alleles of EGFR, from the 40 patients with SCLC who were received surgical treatment in this study. SurPlex-xTAG was adapted and standardized by Sur-Exam (Guangzhou, China) based on the $\mathrm{xTAG}^{\circledast}$ technology by Luminex (Luminex Corp., TX, USA). This method is qualitative. When the MFI is above the cut-off value, the sample is defined as positive. Recently, a number of methods have been applied for somatic mutation screening. These methods include single strand conformation polymorphism (SSCP), scorpions amplified refractory mutation system (scorpions ARMS) and mutant-enriched PCR [10]. In comparison, the main advantage of the SurPlex-xTAG is that it is high throughput and allows for the detection of multiple alleles in one reaction tube from one FFPE slide. Also, up to 96 samples (including one negative and one positive control) could be analyzed in one day. It is very sensitive, robust, and easily standardized for routine clinical testing [9].

EGFR mutations were predictive to response of single-agent TKIs, EGFR gene copy was also associated with response to 
TKIs, albeit with lower sensitivity and specificity [11]. So in our study, we detected the EGFR mutations not EGFR copy.

Better survival were observed in gefitinib-treated NSCLC patients with EGFR exon 19 than exon 21 mutations, gefitinib produced G1 arrest in more of the cells with exon 19 deletion than with L858R[12]. The mutations included two deletions in exon 19: del 2237-2255 insT (del E746-S752 insV) in one patient and del 2235-2249 (del E746-A750) in another patient reported by Shiao TH in Taiwan, and the histologic type of all these two patients are conventional. The SCLCs harboring EGFR mutation were more likely to be combined with adenocarcinoma compared with the whole SCLC population [5]. The mutations included L858R mutations (three patients), a G719A mutation (one patient), and a 15-bp deletion in exon 19 (one patient), reported by Akiko Tatematsu in Japan [5]. In our study, The mutation in exon 19: del E746-A750(K745 AAA) of the EGFR gene is in a male patient which pathology is SCLC combined squamous cell carcinoma, and the other patient is female which pathology is SCLC combined adenocarcinoma.

Based on the 40 cases studied in this report, the incidence of EGFR mutation(5\%) in SCLC is low. In detail, one patient with EGFR exon 19 mutation is female whose pathology is SCLC combined with adenocarcinoma. Notably, in three tumors of the combined SCLC subtype, both components of adenocarcinoma and SCLC harbored an EGFR mutation [5]. In terms of molecular pathogenesis, it is suggested that some SCLCs may have developed from pre-existing adenocarcinomas with EGFR mutations. We think that EGFR mutation might occur more often in combined SCLCs than conventional patients.

Funds. No.Y2110004, Zhejiang Provincial Natural Science Foundation of China. No.2010KYA035, Zhejiang province medical science fund project of China.

\section{References}

[1] OKAMOTO I, ARAKI J, SUTO R et al. EGFR mutation in gefitinib responsive small-cell lung cancer. Annals of Oncology,2006, 17: 1028-1029. http://dx.doi.org/10.1093/annonc/mdj114

[2] ZAKOWSKI MF, LADANYI M, KRIS MG et al. EGFR Mutations in Small-Cell Lung Cancers in Patients Who Have Never Smoked. N Engl J Med, 2006, 355: 213- 215. http:// dx.doi.org/10.1056/NEJMc053610
[3] QIAN JUN, QIN SHU-KUI, TANG QING-QING et al. Gefitinib in patients with advanced refractory small cell lung cancer contemporaneous with superior venacava syndrome. Chinese Clinical Oncology, 2005, 10: 243-244.

[4] SHIAO TH, CHANG YL, YU CJ et al. Epidermal growth factor receptor mutations in small cell lung cancer: a brief report. J Thorac Oncol, 2011, 5: 195-198. http://dx.doi.org/10.1097/ JTO.0b013e3181f94abb

[5] AKIKO TATEMATSU, JUNICHI SHIMIZU, YOSHIKO MURAKAMI et al. Epidermal Growth Factor Receptor Mutations in Small Cell Lung Cancer. Clin Cancer Res, 2008, 14: 60936096. http://dx.doi.org/10.1158/1078-0432.CCR-08-0332

[6] SHIGEMATSU H, LIN L,TAKAHASHI T et al. Clinical and biological features associated with epidermal growth factor receptor gene mutations in lung cancers. J Natl Cancer Inst, 2005, 97: 339-346. http://dx.doi.org/10.1093/jnci/dji055

[7] WU YL, ZHONG WZ, LI LY et al. Epidermal growth factor receptor mutations and their correlation with gefitinib therapy in patients with non-small cell lung cancer: a meta-analysis based on updated individual patient data from six medical centers in mainland China. J Thorac Oncol, 2007, 2: 430-439. http://dx.doi.org/10.1097/01.JTO.0000268677.87496.4c

[8] NICHOLSON SA, BEASLEY MB, BRAMBILLA E et al. Small cell lung carcinoma (SCLC): a clinicopathologic study of 100 cases with surgical specimens. Am J Surg Pathol, 2002, 26: 11841197. http://dx.doi.org/10.1097/00000478-200209000-00009

[9] GUOQIANG LI, XIAODI LUO, JIAYING HE et al. A novel liquidchip platform for simultaneous detection of 70 alleles of DNA somatic mutations on EGFR, KRAS, BRAF and PIK3CA from formalin-fixed and paraffin-embedded slides containing tumor tissue. Clin Chem Lab Med, 2011, 49: 191-195. http://dx.doi.org/10.1515/CCLM.2011.040

[10] PAO W, LADANYI M. Epidermal growth factor receptor mutation testing in lung cancer: searching for the ideal method. Clin Cancer Res, 2007, 13: 4954-4955. http://dx.doi. org/10.1158/1078-0432.CCR-07-1387

[11] DAHABREH IJ, LINARDOU H, SIANNIS F et al. Somatic EGFR mutation and gene copy gain as predictive biomarkers for response to tyrosine kinase inhibitors in non-small cell lung cancer. Clin Cancer Res, 2010, 16: 291-303. http://dx.doi. org/10.1158/1078-0432.CCR-09-1660

[12] ZHU JQ, ZHONG WZ, ZHANG GC et al. Better survival with EGFR exon 19 than exon 21 mutations in gefitinib-treated non-small cell lung cancer patients is due to differential inhibition of downstream signals. Cancer Lett, 2008, 265: 307- 317. http://dx.doi.org/10.1016/j.canlet.2008.02.064 